\title{
Water Wave Solutions of the Coupled System Zakharov-Kuznetsov and Generalized Coupled KdV Equations
}

\author{
A. R. Seadawy ${ }^{1,2}$ and K. El-Rashidy ${ }^{2,3}$ \\ ${ }^{1}$ Mathematics Department, Faculty of Science, Taibah University, Al-Ula 41921-259, Saudi Arabia \\ ${ }^{2}$ Mathematics Department, Faculty of Science, Beni-Suef University, Beni-Suef, Egypt \\ ${ }^{3}$ Mathematics Department, College of Arts and Science, Taif University, Ranyah, Saudi Arabia
}

Correspondence should be addressed to A. R. Seadawy; aly742001@yahoo.com

Received 19 July 2014; Revised 8 September 2014; Accepted 9 September 2014; Published 12 October 2014

Academic Editor: Praveen Agarwal

Copyright (C) 2014 A. R. Seadawy and K. El-Rashidy. This is an open access article distributed under the Creative Commons Attribution License, which permits unrestricted use, distribution, and reproduction in any medium, provided the original work is properly cited.

An analytic study was conducted on coupled partial differential equations. We formally derived new solitary wave solutions of generalized coupled system of Zakharov-Kuznetsov (ZK) and KdV equations by using modified extended tanh method. The traveling wave solutions for each generalized coupled system of $\mathrm{ZK}$ and $\mathrm{KdV}$ equations are shown in form of periodic, dark, and bright solitary wave solutions. The structures of the obtained solutions are distinct and stable.

\section{Introduction}

Many nonlinear evolution equations are playing important role in the analysis of some phenomena. In the study of equations modeling wave phenomena, one of the fundamental objects is the traveling wave solution. Traveling wave solution expressions are in explicit or implicit forms. These types of waves will not change their shapes during propagation. The particular interests are three types of traveling waves: the solitary waves, which are localized traveling waves, asymptotically zero at large distances, the periodic waves, and the kink waves, which rise or descend from one asymptotic state to another. A unified method, called the extended mapping method, is developed to obtain exact traveling wave solutions for a large variety of nonlinear partial differential equations $[1,2]$. By means of this method, the solitary wave, the periodic wave, and the kink wave solutions can be obtained simultaneously. In order to describe complex phenomena in various fields of science, some important nonlinear evolution equations have been established, such as Kadomtsev Petviashvili (KP) equation, Korteweg-de Vries (KdV) equation, and Zakharov-Kuznetsov (ZK) equation [3]. The KdV equation is a model to describe and identify mechanisms for atmospheric blocking. The ZK equation governs the behavior of weakly nonlinear ion-acoustic waves in plasma comprising cold ions and hot isothermal electrons in the presence of a uniform magnetic field [4, 5]. Moreover, ZK equation supports stable solitary waves, which makes ZK equation a very attractive model equation for the study of vortices in geophysical flows $[5,6]$.

The ZK equation was first derived for describing weakly nonlinear ion-acoustic waves in strongly magnetized lossless plasma in two dimensions [4]. Wazwaz [7] used extended tanh method for analytic treatment of the $\mathrm{ZK}$ equation, the modified $\mathrm{ZK}$ equation, and the generalized forms of these equations. Huang [8] applied the polynomial expansion method to solve the coupled ZK equations. Zhao et al. [9] obtained numbers of solitary waves, periodic waves, and kink waves using the theory of bifurcations of dynamical systems for the modified ZK equation. Inc [10] solved nonlinear dispersive $\mathrm{ZK}$ equations using the Adomian decomposition method, and Biazar et al. [11] applied the homotopy perturbation method to solve the ZK equations. In [12], the approximate analytical solution of a Zakharov-Kuznetsov ZK $(m, n$, $k$ ) equation with the help of the differential transform method (DTM) is presented. The DTM method is a powerful and efficient technique for finding solutions of nonlinear equations without the need of a linearization process. The DTM 
is an analytical method based on a Taylor expansion. This method constructs an analytical solution in the form of a polynomial [13-15]. The application of DTM is successfully extended to obtain analytical approximate solutions to various linear and nonlinear problems $[16,17]$.

The coupled KdV system, since Hirota and Satsuma presented the first coupled KdV system [18], its properties have been researched amply [19-21]. After that, a series of important coupled KdV models are constructed [22]. Some kinds of general coupled $\mathrm{KdV}$ equations gain real application in some fields such as in shallow stratified liquid [23-25], atmospheric dynamical system [26], and two-component Bose-Einstein condensates [27]. Lou et al. [28] obtained exact solutions of a coupled $\mathrm{KdV}$ system with a formally variable separation approach and derived a coupled variable coefficient $\mathrm{mKdV}$ equation from a two-layer fluid system [29]. Hu et al. [30] discovered nonsingular positon and complexiton solutions for a special coupled KdV system by means of the iterative Darboux transformation. The research about solution, structure, interaction, and other properties of soliton abstracts much more attention and many meaningful results are obtained successfully [31-35].

This paper is organized as follows. An introduction in Section 1. In Section 2, the formulation of stability analysis solutions. In Section 3, we found the exact soliton solutions for the coupled system of $\mathrm{ZK}$ equations. The travelling wave solutions of the generalized system of $\mathrm{KdV}$ equations are obtained in Section 4. Finally, the paper end with a conclusion in Section 5.

\section{Stability of Solutions}

Hamiltonian system for which the momentum is given by

$$
M=\frac{1}{2} \iint_{-\infty}^{\infty} U_{i j}^{2}(t, x) d t d x, \quad i=1,2, \quad j=1,2,3,
$$

where $U_{1}=u(x, t)$ and $U_{2}=v(x, t)$. The sufficient condition for discussing the stability of solution $\partial M / \partial k>0$.

\section{The Generalized Coupled ZK Equations}

The general forms of the coupled ZK equations [8] are

$$
\begin{gathered}
u_{t}+u_{x x x}+u_{y y x}-6 u u_{x}-v_{x}=0, \\
v_{t}+\delta v_{x x x}+\lambda v_{y y x}+\eta v_{x}-6 \mu \nu v_{x}-\alpha u_{x}=0 .
\end{gathered}
$$

The coupled ZK equations are the model describing two interacting weakly nonlinear waves in anisotropic background stratified followed flows. Here, $x$ and $y$ are the propagation and transverse coordinates, $\eta$ is a group velocity shift between the coupled models, $\delta$ and $\lambda$ are the relative longitudinal and transverse dispersion coefficient, and $\mu$ and $\alpha$ are the relative nonlinear and coupled coefficients. In case $\left[u_{y}=v_{y}=0\right]$, this system reduces to the set of coupled $\mathrm{KdV}$ equations. To look for the traveling wave solutions of coupled ZK equation (2). Consider the traveling wave solutions:

$$
\begin{gathered}
u(x, y, t)=U(\xi)=\sum_{i=0}^{m} a_{i} \varphi^{i}(\xi)+\sum_{i=1}^{m} c_{i} \varphi^{-i}(\xi) \\
v(x, y, t)=V(\xi)=\sum_{j=0}^{n} b_{j} \varphi^{j}(\xi) \sum_{j=1}^{n} d_{j} \varphi^{-j}(\xi) \\
\frac{d \varphi}{d \xi}=\sqrt{\varphi^{2}-\varphi^{4}}, \quad \xi=k x+\nu y+\omega t
\end{gathered}
$$

where $a_{i}, c_{i}, b_{j}, d_{j}, v, k$, and $\omega$ are arbitrary constants and $m$ and $n$ are positive integers, in most cases, that will be determined. The parameters $m$ and $n$ are usually obtained by balancing the linear terms of the highest order in the resulting equation with the highest order nonlinear terms. Substituting (3) into (2), (2) becomes

$$
\begin{gathered}
\left(k^{3}+v^{3}\right) U^{\prime \prime}+(\omega-3 k U) U-k V=0, \\
\left(\delta k^{3}+\lambda k \nu^{2}\right) V^{\prime \prime}+(\omega+k \eta-6 k \mu V) V-\alpha k V=0 .
\end{gathered}
$$

We suppose that the solution of (5) is in the following form:

$$
\begin{aligned}
& U(\xi)=a_{0}+a_{1} \varphi+c_{1} \varphi^{-1}+a_{2} \varphi^{2}+c_{2} \varphi^{-2}, \\
& V(\xi)=b_{0}+b_{1} \varphi+d_{1} \varphi^{-1}+b_{3} \varphi^{2}+d_{2} \varphi^{-2} .
\end{aligned}
$$

Substituting (6) into (5) yields a set of algebraic equations for $a_{0}, a_{1}, a_{2}, c_{1}, c_{2}, b_{0}, b_{1}, b_{2}, d_{1}, d_{2}, \alpha, \delta, \lambda, \nu, \omega, k, \mu$, and $\eta$. We have two cases for these equations that are found as follows.

Case 1. In the solution of the system of (5), we can find

$$
\begin{gathered}
a_{0}=-\frac{\alpha+\eta+\omega}{6 \mu}, \quad a_{1}=-1, \quad a_{2}=c_{2}=0, \\
c_{1}=\frac{3 k(\alpha+2 \mu)}{\alpha(3 k+\omega)}, \\
b_{0}=\frac{\alpha+\eta+\omega}{6 \mu}, \quad b_{1}=-1, \quad b_{2}=d_{2}=0, \\
d_{1}=\frac{3(k \alpha+8 k \mu+2 \mu \omega)}{\alpha(3 k+\omega)} .
\end{gathered}
$$

Substituting (7) and (8) into (6) with $\nu=-k$ and $\delta=-\lambda$, we have obtained the following solutions of (2):

$$
\begin{aligned}
u(x, y, t)= & -\frac{\alpha+\eta+\omega}{6 \mu}-\operatorname{sech}(k x+v y+\omega t) \\
& +\frac{3 k(\alpha+2 \mu)}{\alpha(3 k+\omega)} \operatorname{sech}^{2}(k x+\nu y+\omega t), \\
v(x, y, t)= & \frac{\alpha+\eta+\omega}{6 \mu}-\operatorname{sech}(k x+v y+\omega t) \\
& +\frac{3(k \alpha+8 k \mu+2 \mu \omega)}{\alpha(3 k+\omega)} \operatorname{sech}^{2}(k x+v y+\omega t)
\end{aligned}
$$




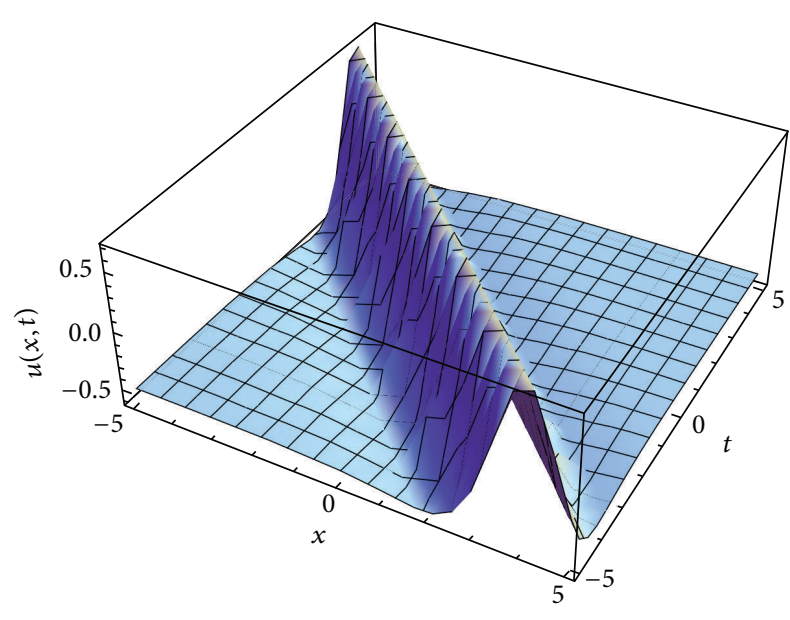

(a)

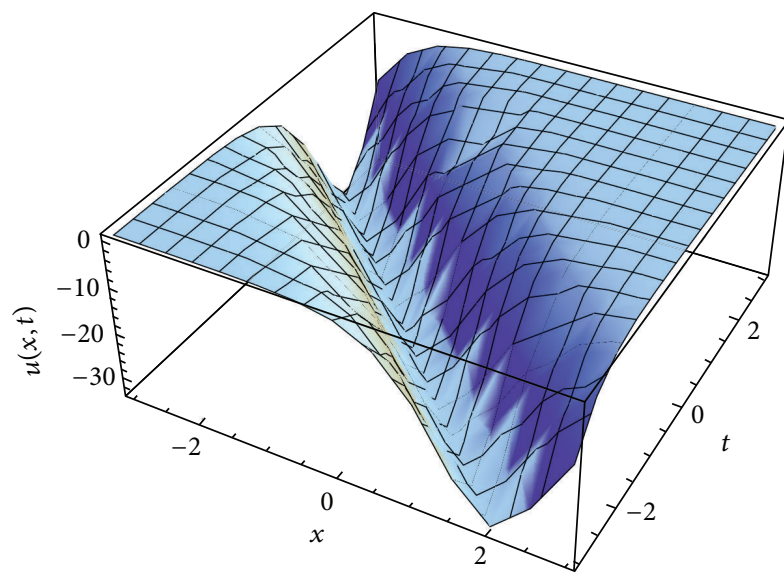

(c)

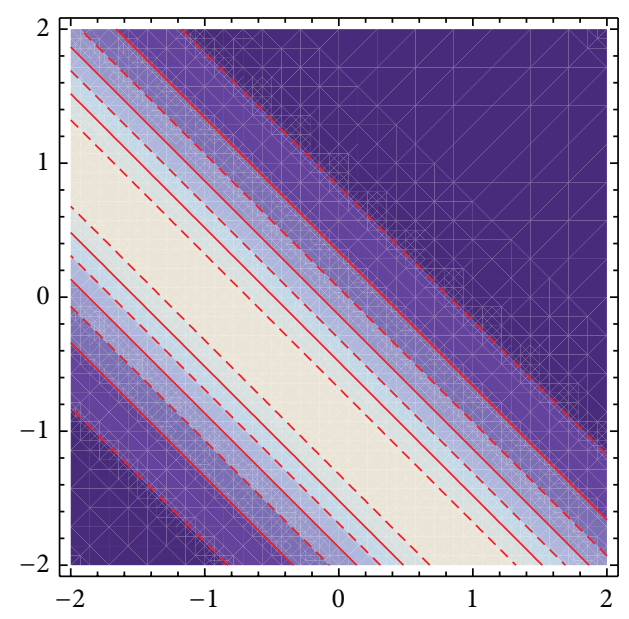

(b)

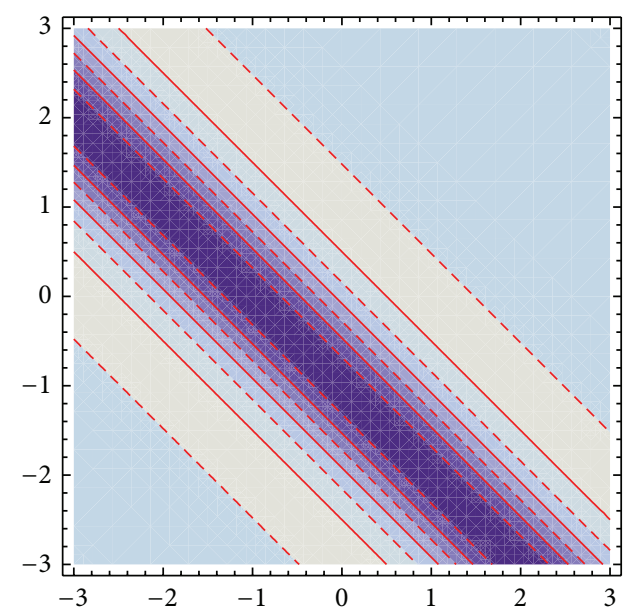

(d)

FIGURE 1: Travelling waves solutions (9) and (10) with various different shapes are plotted: bright solitary waves in (a) and contour plot in (b). Travelling waves solutions (13) and (14) with various different shapes are plotted: dark solitary waves in (c) and contour plot in (d).

Case 2. The solutions of the system of (5) can be found as follows:

$$
\begin{gathered}
a_{0}=\frac{2 \alpha-\eta+\omega}{3 \mu}, \quad a_{1}=2, \quad a_{2}=c_{2}=0, \\
c_{3}=\frac{12 k(\alpha+2 \mu)}{\alpha \omega}, \\
b_{0}=\frac{-2 \alpha+\eta+\omega}{6 \mu}, \quad b_{1}=2, \quad b_{2}=d_{2}=0, \\
d_{1}=\frac{12(k \alpha+2 k \mu+2 \mu \omega)}{\alpha \omega} .
\end{gathered}
$$

Substituting (11) and (12) into (6), we obtained the following solutions of (2):

$$
\begin{aligned}
u(x, y, t)= & \frac{2 \alpha-\eta+\omega}{3 \mu}+2 \operatorname{sech}(k x+\nu y+\omega t) \\
& +\frac{12 k(\alpha+2 \mu)}{\alpha \omega} \operatorname{sech}^{2}(k x+\nu y+\omega t),
\end{aligned}
$$

$$
\begin{aligned}
v(x, y, t)= & \frac{-2 \alpha+\eta+\omega}{6 \mu}+2 \operatorname{sech}(k x+\nu y+\omega t) \\
& +\frac{12(k \alpha+2 k \mu+2 \mu \omega)}{\alpha \omega} \operatorname{sech}^{2}(k x+\nu y+\omega t) .
\end{aligned}
$$

Figures 1(a) and 1(c) represent the evolution of the bright and dark solitary wave solutions (9) and (13) of the generalized coupled system ZK equation (2), with $\alpha=\eta=\omega=$ $\mu=\nu=1$, and $k=-1$. The solitary wave solutions (8)(9) are stable in the intervals $[-5,5]$ and $[-3,3]$. A contour plots Figures 1(b) and 1(d) are a collection of level curves drawn on the same set of axes. The Mathematica command ContourPlot draws contour plots of functions of two variables. The contours join points on the surface having the same height. The default is to have contours corresponding to a sequence of equally spaced values of the function. 


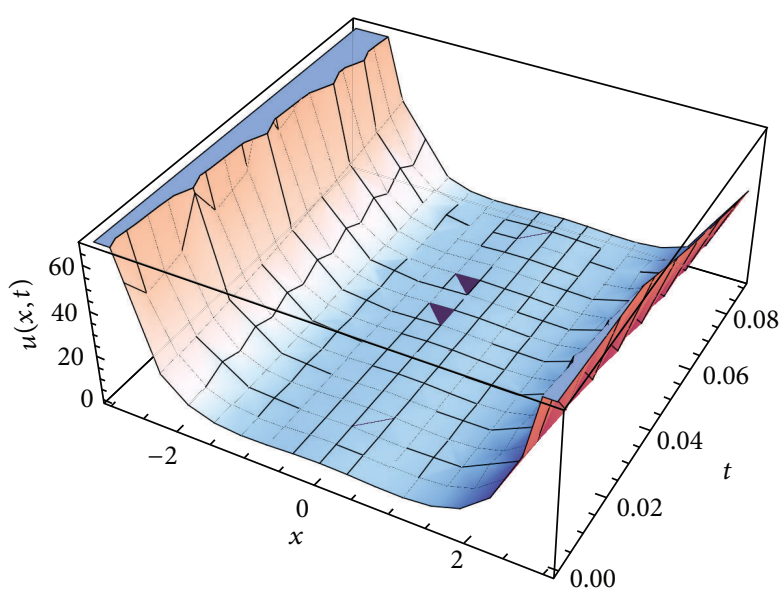

(a)

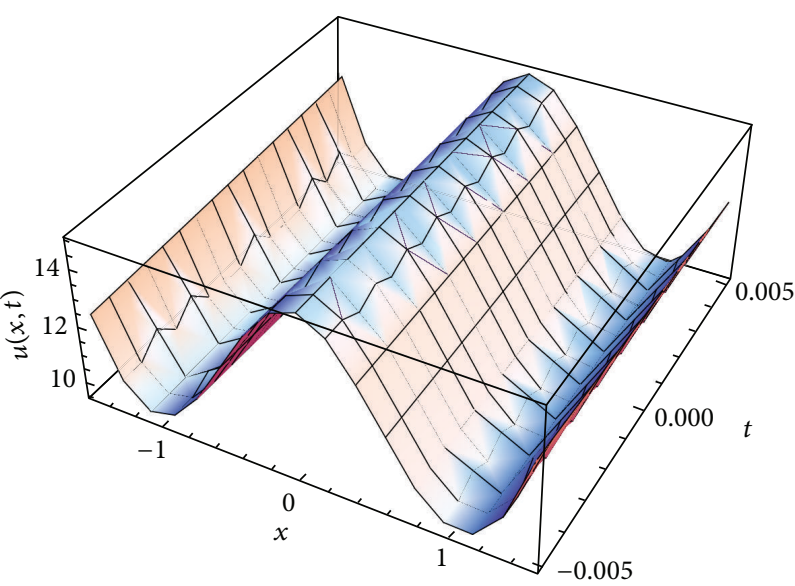

(c)

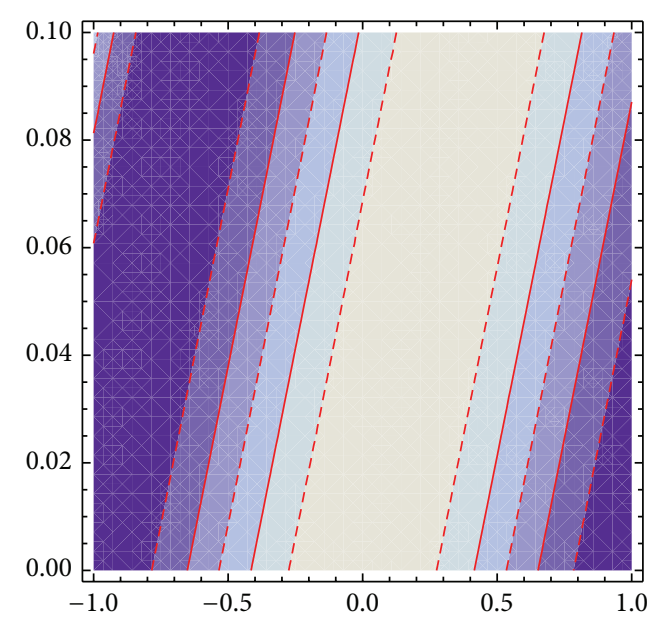

(b)

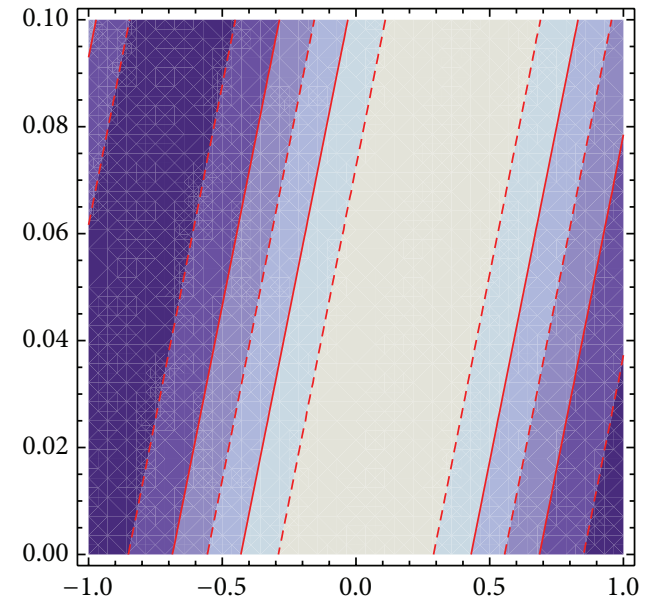

(d)

FIGURE 2: Travelling waves solution (20) with various different shapes are plotted: dark solitary waves in (a) and contour plot in (b). Travelling waves solution (22) with various different shapes are plotted: periodic solitary waves in (d) and contour plot in (c).

\section{The Generalized Coupled KdV Equation}

Consider the following generalized coupled KdV equations:

$$
\begin{aligned}
& u_{t}+\alpha_{1} v u_{x}+\left(\alpha_{2} v^{2}+\alpha_{3} u v+\alpha_{4} u_{x x}+\alpha_{5} u^{2}\right)_{x}=0 \\
& v_{t}+\delta_{1} v u_{x}+\left(\delta_{2} u^{2}+\delta_{3} u v+\delta_{4} v_{x x}+\delta_{5} v^{2}\right)_{x}=0
\end{aligned}
$$

where $\alpha_{i}, \delta_{i}(i=1,2,3,4,5)$ are arbitrary constants. This system is derived from two-layer fluids, whose integrability and existence of the solitarty wave solutions for this system have been discussed by Lou et al. [26]. Let us consider the traveling wave solutions $u(x, t)=U(\xi), v(x, t)=V(\xi)$, and $\xi=x-c t$, and then (15) becomes

$$
\begin{aligned}
& -c U^{\prime}+\alpha_{1} V U^{\prime}+\left(\alpha_{2} V^{2}+\alpha_{3} U V+\alpha_{4} U^{\prime \prime}+\alpha_{5} U^{2}\right)^{\prime}=0 \\
& -c V^{\prime}+\delta_{1} V U^{\prime}+\left(\delta_{2} U^{2}+\delta_{3} U V+\delta_{4} V^{\prime \prime}+\delta_{5} V^{2}\right)^{\prime}=0 .
\end{aligned}
$$

We assume that $\alpha_{1}=\delta_{1}=0$ and $\alpha_{4}=\delta_{4}=1$, so (16) becomes

$$
\begin{aligned}
& -c U+\left(\alpha_{2} V^{2}+\alpha_{3} U V+U^{\prime \prime}+\alpha_{5} U^{2}\right)=0, \\
& -c V+\left(\delta_{2} U^{2}+\delta_{3} U V+V^{\prime \prime}+\delta_{5} V^{2}\right)=0 .
\end{aligned}
$$

Balancing the nonlinear term $U V$ and the highest order derivative $U^{\prime \prime}$ gives $m=2$. We suppose that the solution of (17) is in the forms

$$
\begin{gathered}
U(\xi)=a_{0}+a_{1} \varphi+a_{2} \varphi^{-1}+a_{3} \varphi^{2}+a_{4} \varphi^{-2} \\
V(\xi)=b_{0}+b_{1} \varphi+b_{2} \varphi^{-1}+b_{3} \varphi^{2}+b_{4} \varphi^{-2} .
\end{gathered}
$$

Substituting (18) into (17) yields a set of algebraic equations for $a_{0}, a_{1}, a_{2}, a_{3}, a_{4}, b_{0}, b_{1}, b_{2}, b_{3}$, and $b_{4}$. We have two cases for these equations that are found as follows. 
Case 1. In the solution of the system of (17), we can find

$$
\begin{gathered}
a_{0}=1, \quad a_{1}= \pm \sqrt{\frac{85}{6}}, \quad a_{2}=\mp 9 \sqrt{\frac{3}{170}}, \\
a_{3}=1, \quad a_{4}=\frac{343}{340}, \\
b_{0}=0, \quad b_{1}= \pm \sqrt{510}, \quad b_{2}=0, \\
b_{3}=\frac{97}{6}, \quad b_{4}=\frac{243}{85},
\end{gathered}
$$

with $\alpha_{2}=1, \alpha_{5}=\delta_{5}=6, \alpha_{3}=-6, \delta_{2}=36, \delta_{3}=-36$, and $c=4$.

Substituting (19) into (18), we have obtained the following solutions of (15):

$$
\begin{aligned}
u(x, t)= & 1 \pm \sqrt{\frac{85}{6}} \operatorname{sech}(x-c t) \mp 9 \sqrt{\frac{3}{170}} \cosh (x-c t) \\
& +\operatorname{sech}^{2}(x-c t)+\frac{343}{340} \cosh ^{2}(x-c t), \\
v(x, t)= & \pm \sqrt{510} \operatorname{sech}(x-c t)+\frac{97}{6} \operatorname{sech}^{2}(x-c t) \\
& +\frac{243}{85} \cosh ^{2}(x-c t) .
\end{aligned}
$$

Case 2. In the solution of the system of (12), we find

$$
\begin{gathered}
a_{0}=1, \quad a_{1}=\frac{1}{45}( \pm 4 \sqrt{381} \pm \sqrt{10146}), \\
a_{2}= \pm \sqrt{\frac{127}{75}}, \quad a_{3}=1, \quad a_{4}=\frac{1}{2}, \\
b_{0}=0, \quad b_{1}=\frac{2}{15}( \pm 4 \sqrt{381} \pm \sqrt{10146}), \\
b_{2}=0, \quad b_{3}=16, \quad b_{4}=2 .
\end{gathered}
$$

Substituting (19) into (18), we have obtained the following solutions of (15):

$$
\begin{aligned}
u(x, t)= & 1+\frac{1}{45}( \pm 4 \sqrt{381} \pm \sqrt{10146}) \operatorname{sech}(x-c t) \\
& \pm \sqrt{\frac{127}{75}} \cosh (x-c t)+\operatorname{sech}^{2}(x-c t) \\
& +\frac{1}{2} \cosh ^{2}(x-c t) \\
v(x, t)= & \frac{2}{15}( \pm 4 \sqrt{381} \pm \sqrt{10146}) \operatorname{sech}^{(x-c t)} \\
& +16 \operatorname{sech}^{2}(x-c t)+2 \cosh ^{2}(x-c t) .
\end{aligned}
$$

Figures 2(a) and 2(b) represent the evolution of the dark and periodic solitary wave solutions (20) and (22) of the generalized coupled system $\mathrm{KdV}$ equation (15), with $\alpha_{2}=1$, $\alpha_{5}=\delta_{5}=6, \alpha_{3}=-6, \delta_{2}=36, \delta_{3}=-36$, and $c=4$. The solitary wave solutions (20) and (22) are in the intervals $[0,1]$ and $[-2,2]$.

\section{Conclusion}

The basic goal of this work has been the study of a generalized ZK equations, which is important in mathematics and physics. The explicit solutions of GZK equations, KdV system equations, and $\mathrm{KdV}$ equation are obtained. These exact solutions might provide a useful help for physicists to study more complicated physical phenomena. All soliton solutions are exact and stable and have applications in physics.

\section{Conflict of Interests}

The authors declare that there is no conflict of interests regarding the publication of this paper.

\section{References}

[1] Y.-Z. Peng, "Exact solutions for some nonlinear partial differential equations," Physics Letters A, vol. 314, no. 5-6, pp. 401-408, 2003.

[2] Y.-Z. Peng, "New exact solutions to a new Hamiltonian amplitude equation," Journal of the Physical Society of Japan, vol. 73, no. 5, pp. 1156-1158, 2004.

[3] G. Gottwald and R. Grimshaw, "The formation of coherent structures in the context of blocking," Journal of the Atmospheric Sciences, vol. 56, no. 21, pp. 3640-3662, 1999.

[4] V. E. Zakharov and E. A. Kuznetsov, "On three-dimensional solitons," Soviet Physics, vol. 39, pp. 285-288, 1974.

[5] A. R. Seadawy, "Stability analysis for Zakharov-Kuznetsov equation of weakly nonlinear ion-acoustic waves in a plasma," Computers \& Mathematics with Applications, vol. 67, no. 1, pp. 172-180, 2014.

[6] A. R. Seadawy, "Stability analysis for two-dimensional ionacoustic waves in quantum plasmas," Physics of Plasmas, vol. 21, no. 5, Article ID 052107, 2014.

[7] A. M. Wazwaz, "The extended tanh method for the ZakharovKuznetsov ZK equation, the modified ZK equation, and its generalized forms," Communications in Nonlinear Science and Numerical Simulation, vol. 13, no. 6, pp. 1039-1047, 2008.

[8] W. Huang, "A polynomial expansion method and its application in the coupled Zakharov-Kuznetsov equations," Chaos, Solitons and Fractals, vol. 29, no. 2, pp. 365-371, 2006.

[9] X. Zhao, H. Zhou, Y. Tang, and H. Jia, “Travelling wave solutions for modified Zakharov-Kuznetsov equation," Applied Mathematics and Computation, vol. 181, no. 1, pp. 634-648, 2006.

[10] M. Inc, "Exact solutions with solitary patterns for the ZakharovKuznetsov equations with fully nonlinear dispersion," Chaos, Solitons and Fractals, vol. 33, no. 5, pp. 1783-1790, 2007.

[11] J. Biazar, F. Badpeima, and F. Azimi, "Application of the homotopy perturbation method to Zakharov-Kuznetsov equations," Computers \& Mathematics with Applications, vol. 58, no. 11-12, pp. 2391-2394, 2009. 
[12] X. Zhou, Differential Transformation and Its Applications for Electrical Circuits, Huazhong University Press, Wuhan, China, 1986, (Chinese).

[13] S. Dinarvand, S. Khosravi, A. Doosthoseini, and M. M. Rashidi, "The homotopy analysis method for solving the Sawada-Kotera and Lax's fifth-order KdV equations," Advances in Theoretical and Applied Mechanics, vol. 1, pp. 327-335, 2008.

[14] M. M. Rashidi, G. Domairry, A. DoostHosseini, and S. Dinarvand, "Explicit approximate solution of the coupled KdV equations by using the homotopy analysis method," International Journal of Mathematical Analysis, vol. 2, no. 9-12, pp. 581-589, 2008.

[15] H. Jafari and M. A. Firoozjaee, "Homotopy analysis method for solving KdV equations," Surveys in Mathematics and Its Applications, vol. 5, pp. 89-98, 2010.

[16] M. M. Rashidi and E. Erfani, "Traveling wave solutions of WBK shallow water equations by differential transform method," Advances in Theoretical and Applied Mechanics, vol. 3, pp. 263271, 2010.

[17] J. Biazar and M. Eslami, "Analytic solution for Telegraph equation by differential transform method," Physics Letters A, vol. 374, no. 29, pp. 2904-2906, 2010.

[18] R. Hirota and J. Satsuma, "Soliton solutions of a coupled Korteweg-de Vries equation," Physics Letters A, vol. 85, no. 8-9, pp. 407-408, 1981.

[19] R. Hirota and J. Satsuma, "A coupled KdV equation is one case of the four-reduction of the KP hierarchy," Journal of the Physical Society of Japan, vol. 51, no. 10, pp. 3390-3397, 1982.

[20] R. Dodd and A. Fordy, "On the integrability of a system of coupled KdV equations," Physics Letters A, vol. 89, no. 4, pp. 168-170, 1982.

[21] H. C. Hu and Q. P. Liu, "New Darboux transformation for Hirota-Satsuma coupled KdV system," Chaos, Solitons and Fractals, vol. 17, no. 5, pp. 921-928, 2003.

[22] M. V. Foursov, "On integrable coupled KdV-type systems," Inverse Problems, vol. 16, no. 1, p. 259, 2000.

[23] J. A. Gear and R. Grimshaw, "Weak and strong interactions between internal solitary waves," Studies in Applied Mathematics, vol. 70, no. 3, pp. 235-258, 1984.

[24] J. A. Gear, "Strong interactions between solitary waves belonging to different wave modes," Studies in Applied Mathematics, vol. 72, no. 2, pp. 95-124, 1985.

[25] A. R. Seadawy and K. El-Rashidy, "Traveling wave solutions for some coupled nonlinear evolution equations," Mathematical and Computer Modelling by using the direct algebraic method, vol. 57, no. 5-6, pp. 1371-1379, 2013.

[26] S. Y. Lou, B. Tong, H.-C. Hu, and X.-Y. Tang, "Coupled KdV equations derived from two-layer fluids," Journal of Physics A: Mathematical and General, vol. 39, no. 3, pp. 513-527, 2006.

[27] V. A. Brazhnyi and V. V. Konotop, "Stable and unstable vector dark solitons of coupled nonlinear Schrödinger equations: application to two-component Bose-Einstein condensates," Physical Review E: Statistical, Nonlinear, and Soft Matter Physics, vol. 72, no. 2, 2005.

[28] S. Y. Lou, X. Y. Tang, and J. Lin, "Exact solutions of the coupled KdV system via a formally variable separation approach," Communications in Theoretical Physics, vol. 36, no. 2, pp. 145-148, 2001.

[29] Y. Gao and X.-Y. Tang, "A coupled variable coefficient modified $\mathrm{KdV}$ equation arising from a two-layer fluid system," Communications in Theoretical Physics, vol. 48, no. 6, pp. 961-970, 2007.
[30] H. C. Hu, B. Tong, and S. Y. Lou, "Nonsingular positon and complexiton solutions for the coupled KdV system," Physics Letters A, vol. 351, no. 6, pp. 403-412, 2006.

[31] M. A. Helal and A. R. Seadawy, "Variational method for the derivative nonlinear Schrödinger equation with computational applications," Physica Scripta, vol. 80, no. 3, Article ID 035004, 2009.

[32] M. A. Helal and A. R. Seadawy, "Exact soliton solutions of a D-dimensional nonlinear Schrödinger equation with damping and diffusive terms," Zeitschrift für Angewandte Mathematik und Physik, vol. 62, no. 5, pp. 839-847, 2011.

[33] A. R. Seadawy, "New exact solutions for the KdV equation with higher order nonlinearity by using the variational method," Computers \& Mathematics with Applications, vol. 62, no. 10, pp. 3741-3755, 2011.

[34] A. R. Seadawy, "Exact solutions of a two-dimensional nonlinear Schrödinger equation," Applied Mathematics Letters, vol. 25, no. 4, pp. 687-691, 2012.

[35] M. A. Helal and A. R. Seadawy, "Benjamin-Feir instability in nonlinear dispersive waves," Computers \& Mathematics with Applications, vol. 64, no. 11, pp. 3557-3568, 2012. 


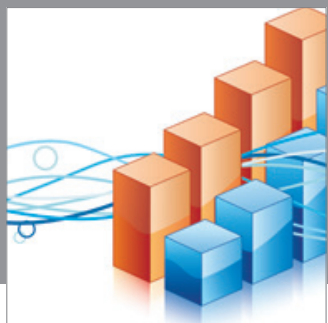

Advances in

Operations Research

mansans

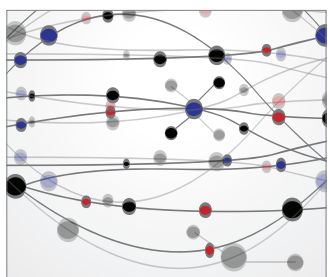

The Scientific World Journal
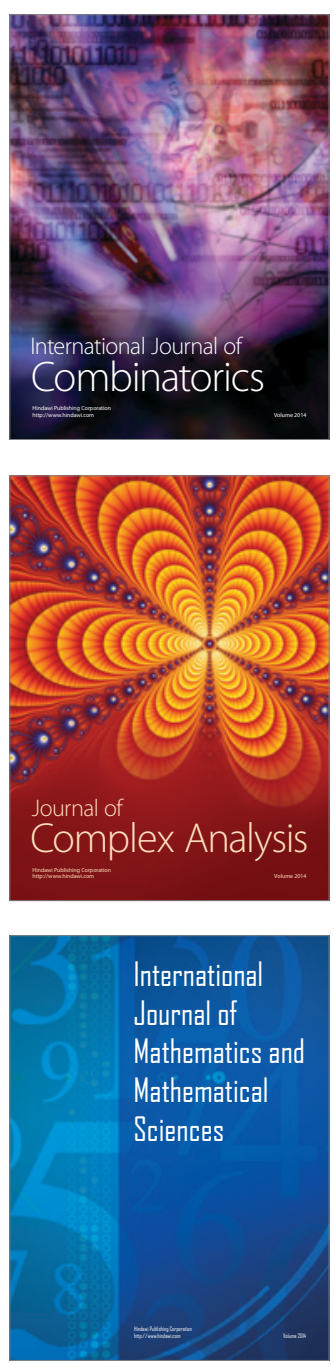
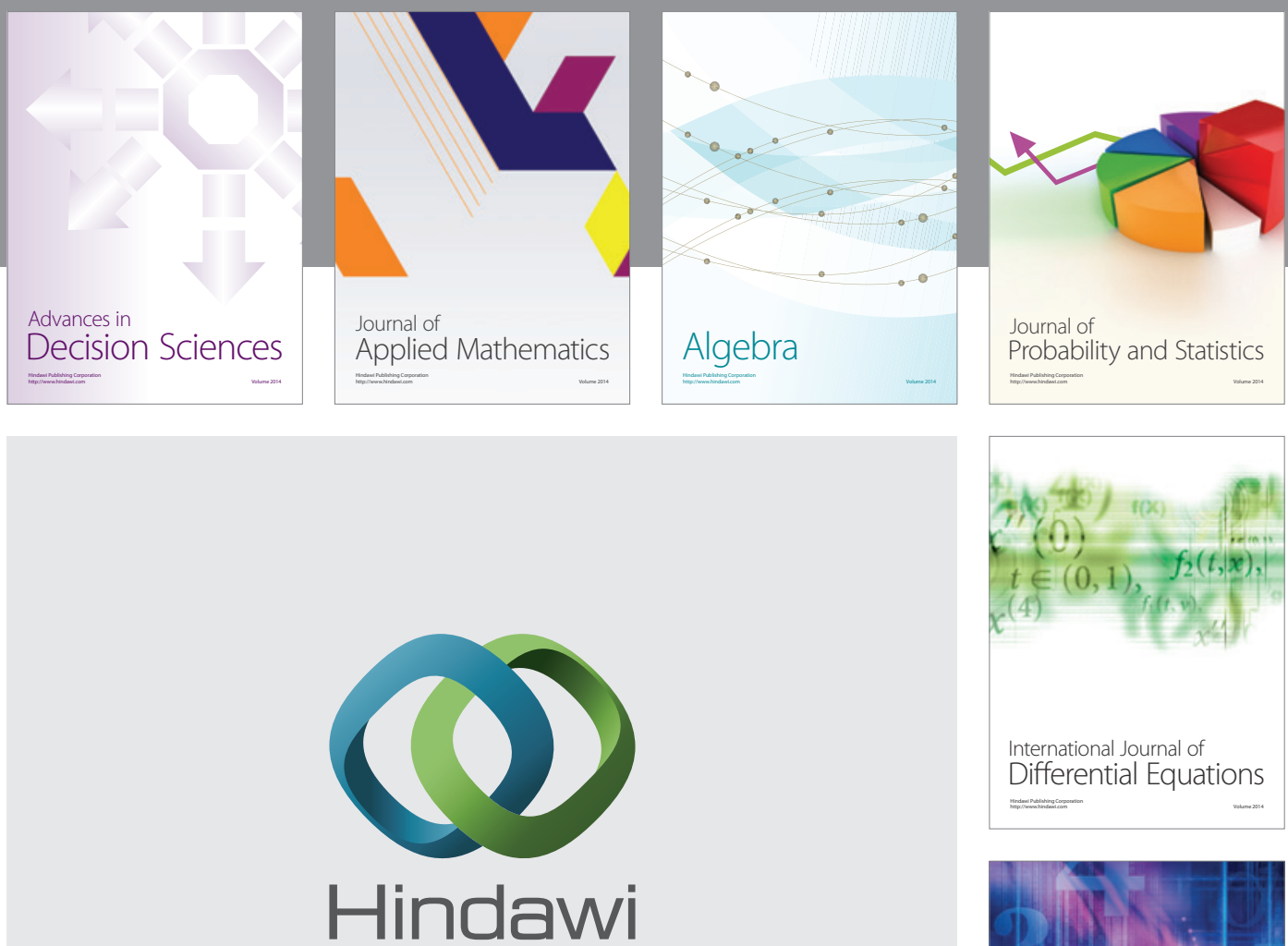

Submit your manuscripts at http://www.hindawi.com
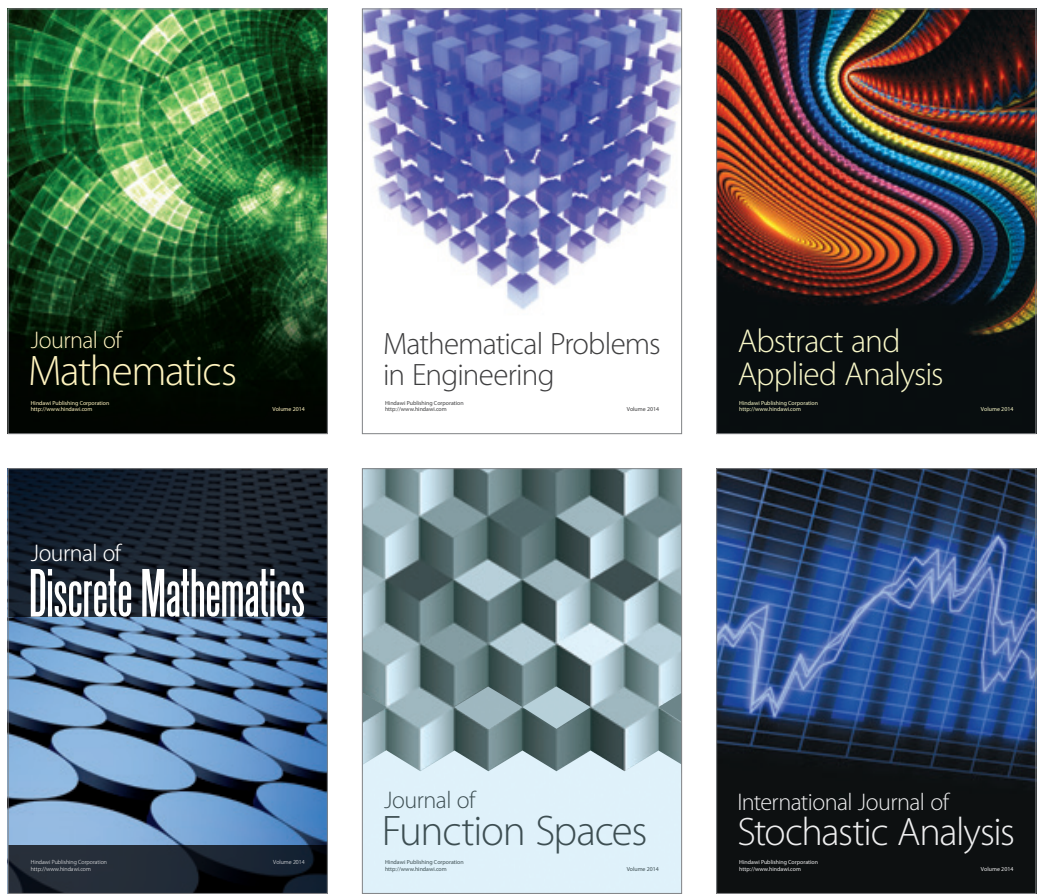

Journal of

Function Spaces

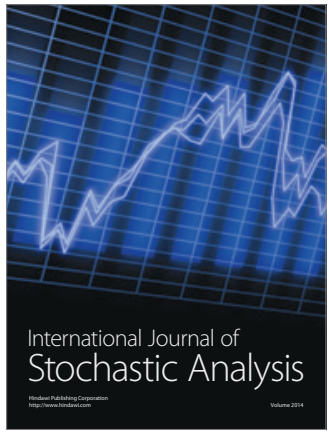

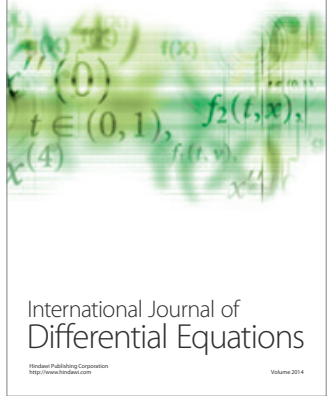
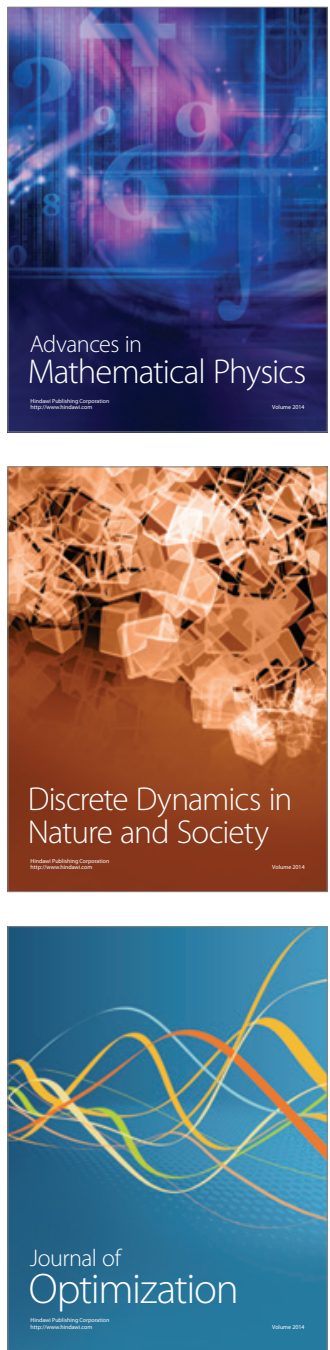